EPJ Web of Conferences 114, 02120 (2016)

DOI: $10.1051 /$ epjconf/201611402120

(C) Owned by the authors, published by EDP Sciences, 2016

\title{
Comparison of two inlet boundary approaches in numerical simulation of car ventilation outlet flow
}

\author{
Tomáš Talanda $^{1 \mathrm{a}}$, Pavel Niedoba ${ }^{1}$, František Lízal $^{1}$ and Miroslav Jícha ${ }^{1}$ \\ ${ }^{1}$ Brno University of Technology, Faculty of Mechanical Engineering, Technická 2, 61669 Brno
}

\begin{abstract}
The paper is concerned with the comparison of two inlet boundary approaches of numerical simulations. The first approach is based on the simulation of a sufficiently long duct upstream the area of interest with known volumetric flow rate. The second approach rests on the experimental measurement of the section closer to the area of interest. The experimental measurement provides velocity profile and in addition turbulent intensity compared to the first approach where only a velocity profile can be computed according to known volumetric flow rate. A simplified model of car ventilation outlet was chosen as a test case. The model consists of circular and rectangular duct, area of interest (closing flap, vertical slats and horizontal slats) and outlet box. We have compared the two mentioned inlet boundary approaches for two distinct values of volumetric flow rate for which the experimental data of the section upstream of the area of interest are available. The velocity and the turbulent kinetic energy profiles downstream of the area of interest were chosen as comparative characteristics.
\end{abstract}

\section{Outline}

The development in computer technology still increases the advantage of numerical simulations compared to experimental measurements. The advantage is meant in the sense of time savings. The experimental measurements are still necessary for tuning of numerical models.

Computational fluid dynamics (CFD) is a tool for simulation of fluid flow [1]. The simulations can be divided into two categories. The first involves simulations which solve Navier-Stokes equations directly, e.g. Direct Numerical Simulation (DNS) and Large Eddy Simulation (LES). Due to enormous computational demands, these methods are still unsuitable for most engineering applications. More feasible is the second category of Reynolds Averaged Navier-Stokes (RANS) simulations which solve timeaveraged Navier-Stokes equations and has to be closed with a turbulence model [2]. The most popular turbulence model for a wide area of applications is k- $\omega$ SST model of Menter [3].

\section{Methodology}

As a computation method for the CFD simulations, RANS simulation closed with the k- $\omega$ SST turbulence model was chosen. This turbulence model combines the $\mathrm{k}-\omega$ turbulence model and $\mathrm{k}-\varepsilon$ turbulence model (where $k$ is turbulent kinetic energy, $\varepsilon$ is turbulent dissipation, and $\omega$ is specific dissipation rate) such that the k- $\omega$ model is used in the inner region of the boundary layer and switches into the $\mathrm{k}-\varepsilon$ model in the free shear flow region. A special blending function based on rates of turbulent kinetic energy, cell size, specific dissipation and viscosity is used for the switching. In addition, the k- $\omega$ turbulence model is suitable mostly for internal flows, while the $\mathrm{k}-\varepsilon$ turbulence model fits well to external flows [1]. In our case, internal flow occurs in the pipe, whereas free shear flow is present downstream of the ventilation outlet.

\section{Results}

In this section, the test cases as well as the numerical setup are described. Also the identification sign of each case is introduced for the comparison purpose in the last part of this section.

\subsection{Test case}

The benchmark ventilation outlet with one closing flap and two rows of slats was chosen as a test case (see figure 2). Airflow through this ventilation outlet was experimentally investigated by Šeda [4].

Altogether four subcases of different pipe geometries (short and long) and flow rates $\left(33.5 \mathrm{~m}^{3} / \mathrm{h}\right.$ and $80.6 \mathrm{~m}^{3} / \mathrm{h}$ ) were investigated (see table 1).

\footnotetext{
${ }^{\mathrm{a}}$ Corresponding author: t.talanda@seznam.cz
} 
Table 1. Case identification sign.

\begin{tabular}{|c|c|c|c|}
\hline \multicolumn{2}{|c|}{} & \multicolumn{2}{c|}{ Flow rate } \\
\cline { 3 - 4 } \multicolumn{2}{|c|}{} & $33.5 \mathrm{~m}^{3} \mathrm{~h}^{-1}$ & $80.6 \mathrm{~m}^{3} \mathrm{~h}^{-1}$ \\
\hline \multirow{3}{*}{ Duct } & Long & $\mathbf{L 3 3}$ & $\mathbf{L 8 0}$ \\
\cline { 2 - 4 } & Short & $\mathbf{S 3 3}$ & $\mathbf{S 8 0}$ \\
\hline
\end{tabular}

\subsubsection{Long duct geometry}

The geometry of the long duct is displayed in the figure 1. It consists of circular duct, on which the rectangular part is connected. At the end of the rectangular duct, the area of interest with flap and slats is situated. The overall length of the duct is $1280 \mathrm{~mm}$ [4]. The outlet part of the geometry is modelled by a sufficiently large box representing free space.

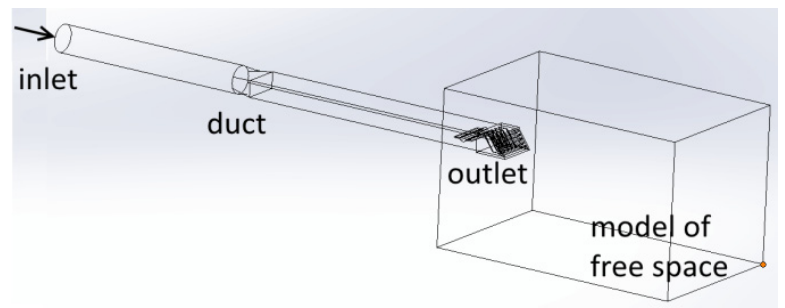

Figure 1. Long duct geometry.

\subsubsection{Short duct geometry}

The geometry of the short duct is depicted in figure 2, as well as the duct dimensions and flap and slats positions. In this case, the duct was shortened to $276 \mathrm{~mm}$ [4].

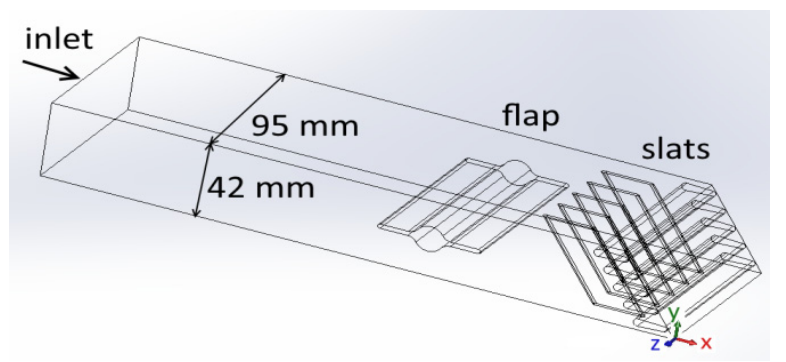

Figure 2. Short duct geometry, flap and slats position.

\subsection{Numerical setup}

The CFD simulation was performed with open source CFD software package OpenFOAM, where the RANS simpleFoam solver was selected. As a turbulence model, the kOmegaSST model was applied using the following wall functions, i.e., kqRWallFunction, nutkWallFunction and omegaWallFunction.

\subsubsection{Cases S33 and S80}

In cases S33 and S80 (see table 1), inlet values of velocity, turbulent kinetic energy $k$ and specific dissipation rate $\omega$ at discrete points were set up according to Šeda experimental data [4]. The values of $k$ and $\omega$ had to be calculated from the experimentally measured root mean square turbulent velocity according to the following formulas:

turbulent kinetic energy $\quad k=\frac{3}{2}\left(u_{R M S}^{\prime}\right)^{2}$

specific dissipation rate $\quad \omega=\frac{\sqrt{k}}{0.038 D_{h}}$

hydraulic diameter

$$
D_{h}=\frac{2 a b}{a+b}
$$

where $a$ and $b$ are channel dimensions.

\subsection{Cases $L 33$ and $L 80$}

In these cases, uniform inlet profiles of velocity, turbulent kinetic energy and specific dissipation rate were prescribed. The calculation of the inlet velocity profile was based on experimentally measured volumetric flow rate. The turbulent kinetic energy and specific dissipation rate were calculated according to the formulas 1 and 2 with an assumption of turbulence intensity $I=14 \%$ $\left(u_{R M S}^{\prime}=\bar{u} I\right)$.

\subsubsection{Computational mesh}

A slice of the computational mesh in the vertical symmetry plane is shown in figure 3 .

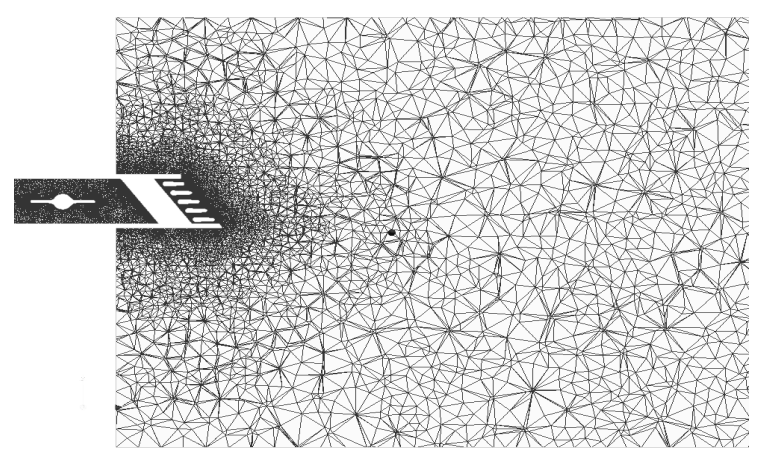

Figure 3. A slice of the computational mesh.

The outlet part was simulated by a large box (figure 1), using zeroGradient boundary condition. The computation mesh was created with an open source Salome mesher containing 4,238,467 tetrahedral cells for cases S33 and S80, and 6,682,931 cells for cases L33 and L80. Parallel computation was performed using a cluster of 32 processors. As a convergence criterion, the $10^{-4}$ residual was considered.

\subsection{Results comparison}

The computational results were compared along lines 1, 2 , and 3 (see figure 4), where line 1 is parallel with the axis of the duct and line 2 represents the axis of the outflow. Line 3 is placed perpendicularly to the duct axis and $0.1 \mathrm{~m}$ downstream from the lower trailing edge of the outlet. 


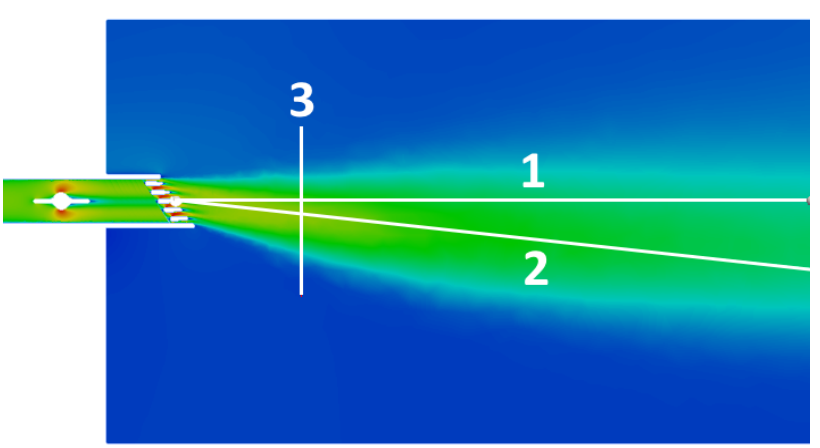

Figure 4. Lines for the results comparison.

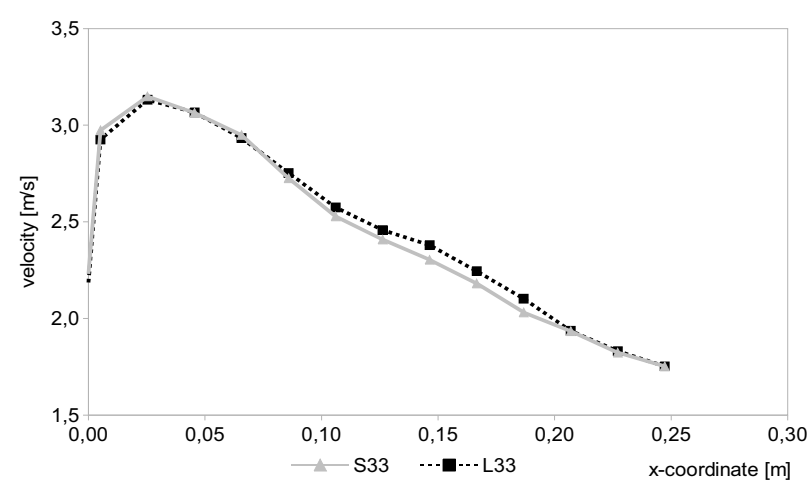

Figure 5. Plot of axial velocity in cases S33 and L33 over line 1

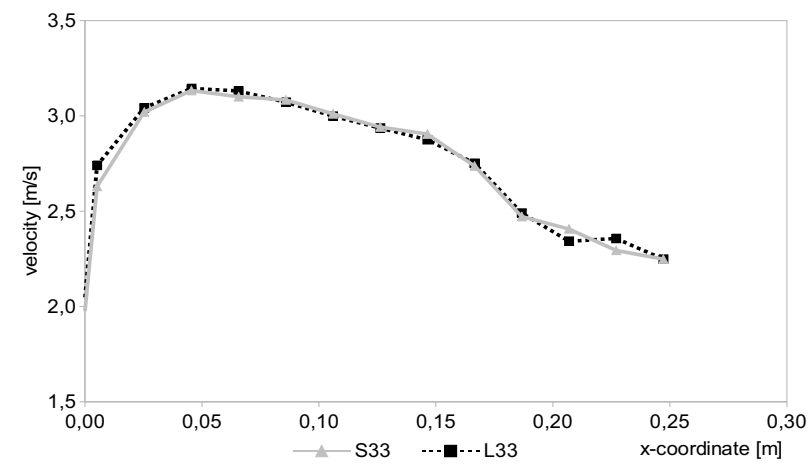

Figure 6. Plot of axial velocity in cases S33 and L33 over line 2

The comparison of axial velocity profiles are shown in figures 5- 7 for the cases S33 and L33 over lines $1-3$. The same is shown for the cases S80 and L80 in figures $8-10$

The comparison of turbulent kinetic energy $k$ profiles for the cases S33 and L33 over lines 1 and 2 can be seen in figures 11 and 12, and for the cases S80 and L80 in figures 13 and 14 .

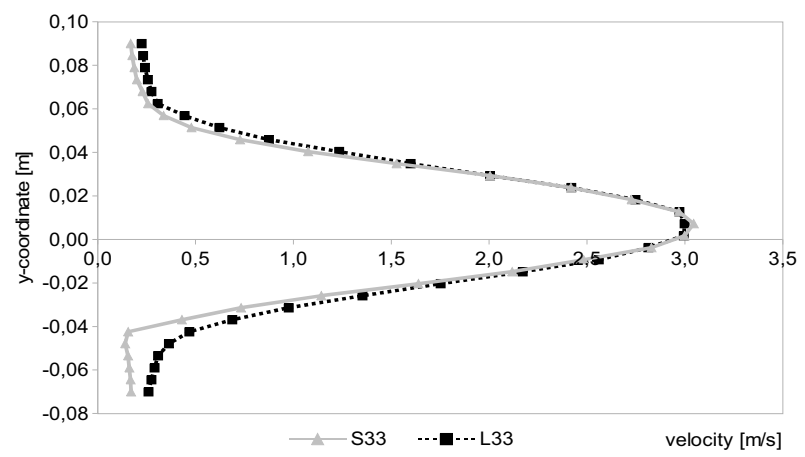

Figure 7. Plot of axial velocity in cases S33 and L33 over line 3

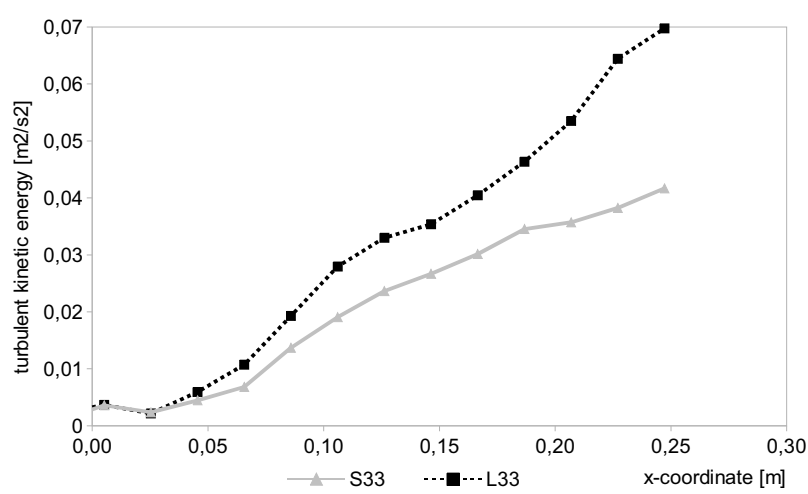

Figure 8. Plot of turbulent kinetic energy $k$ in cases S33 and L33 over line 1.

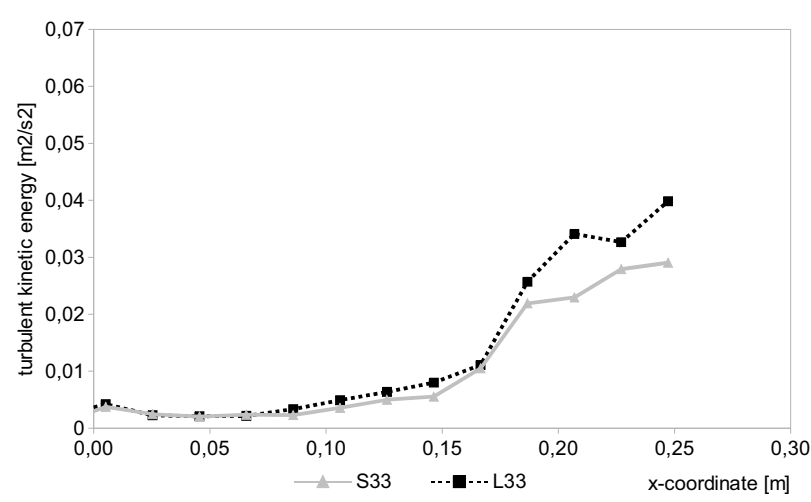

Figure 9. Plot of turbulent kinetic energy $k$ in cases $\mathrm{S} 33$ and L33 over line 2.

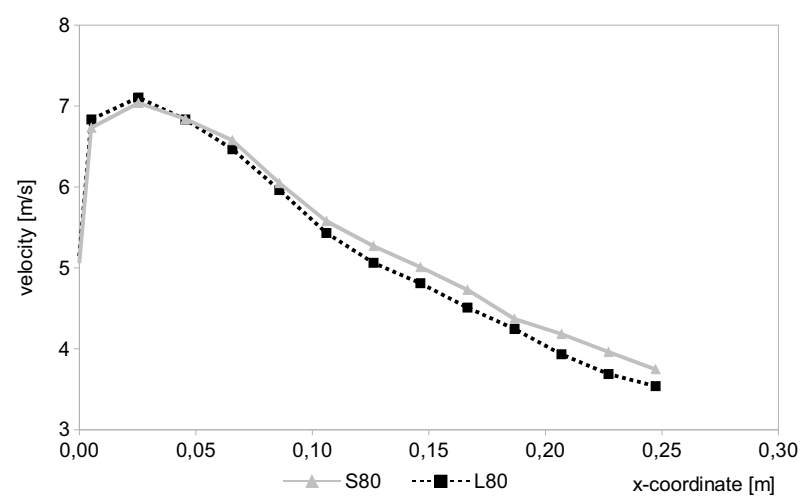

Figure 10. Plot of axial velocity in cases S80 and L80 over line 1. 


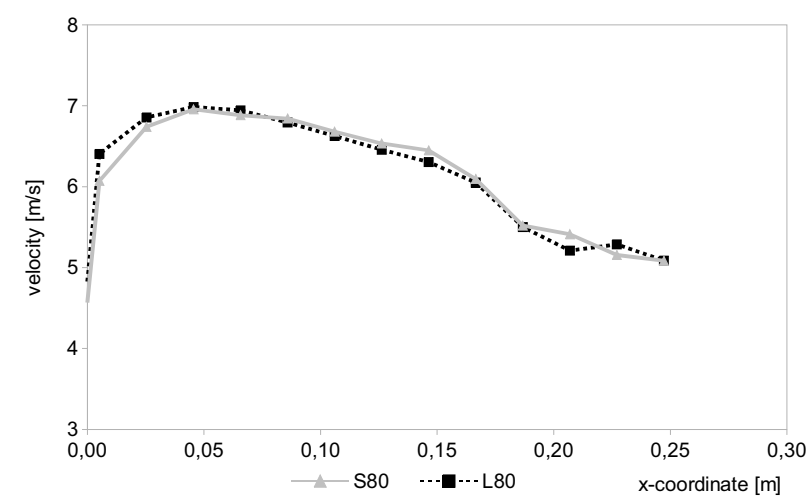

Figure 11. Plot of axial velocity in cases S80 and L80 over line 2 .

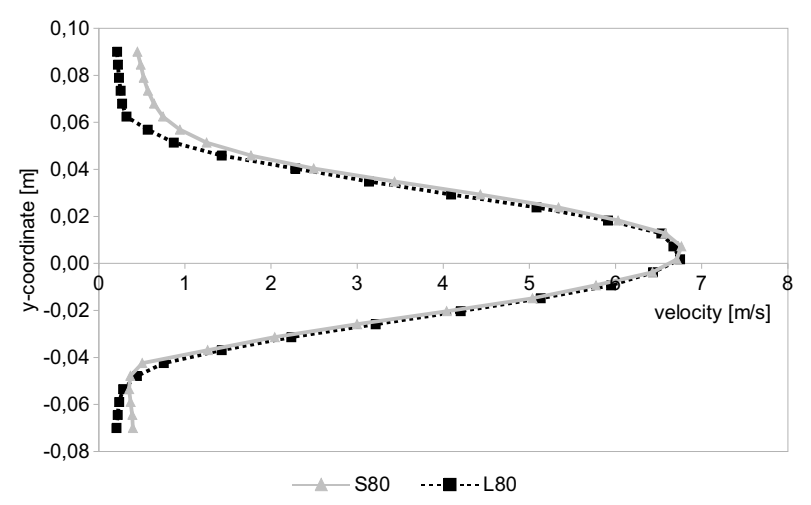

Figure 12. Plot of axial velocity in cases S80 and L80 over line 3.

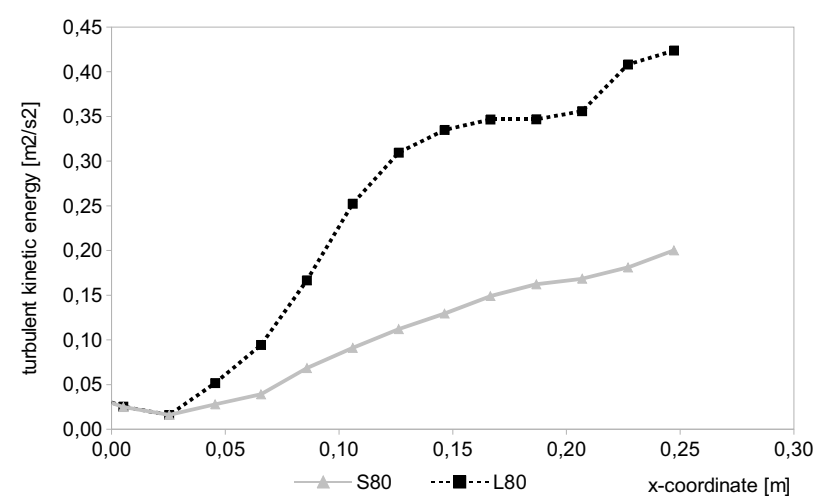

Figure 13. Plot of turbulent kinetic energy $k$ in cases S80 and L80 over line 1.

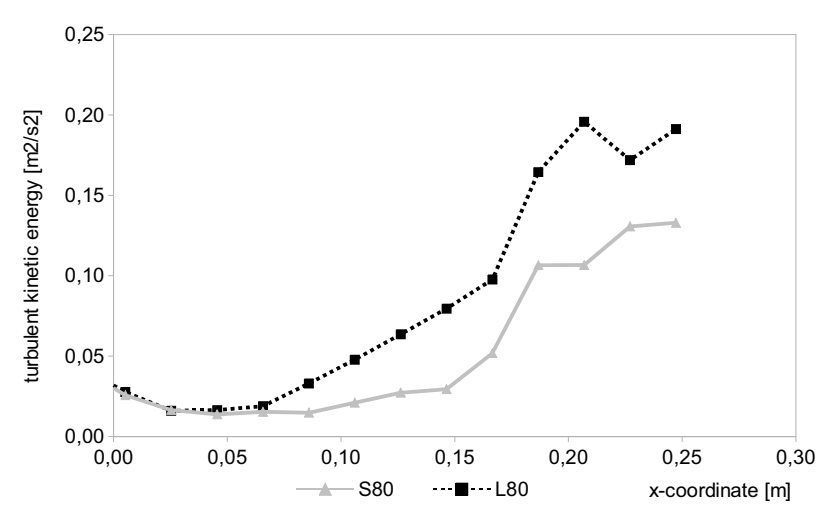

Figure 14. Plot of turbulent kinetic energy $k$ in cases S80 and L80 over line 2.

\section{Conclusion}

In this paper, the axial velocity and the turbulent kinetic energy profiles downstream of the area of interest were compared for two different inlet boundary approaches and two distinct values of volumetric flow rate. The first approach takes into account a sufficiently long duct upstream the area of interest unlike the second approach where the inlet boundary profiles close to the area of interest in upstream direction were provided by experimental measurements. For the first approach, the inlet turbulent kinetic energy had to be computed because only the volumetric flow rate was known.

Resulted axial velocity profiles downstream of the area of interest are in very good agreement. As expected, the slight differences can be seen in the turbulent kinetic energy profiles. The differences may be caused according to the computation of inlet turbulent kinetic energy in the case of the first approach. Despite of the simple geometry, the computation of inlet turbulent kinetic energy has a significant impact on the downstream turbulent kinetic energy.

Thus, for the correct examination of the flow behaviour in the area of interest and downstream of this area, the experimentally measured inlet data sufficiently close to this area are desired. In addition, the first approach requires more computational performance relating to the extended geometry upstream of the area of interest.

\section{Acknowledgement}

This research was supported by the Czech Grant Agency under the grant GA 13-27505S and by the project FV 1519 of the Faculty of Mechanical Engineering at Brno University of Technology.

\section{References}

1. S. B. Pope, Turbulent Flows, Cambridge University Press, 11th printing (2011). ISBN 0521598869

2. H. K. Versteeg and W. Malalasekera, An introduction to computational fluid dynamics: The finite volume method, Pearson Education Ltd., Harlow, UK, 520p (2007).

3. F. R. Menter, AIAA journal 32 (8), 1598 - 1605 (1994).

4. L. Šeda, Analysis of flow from the car vent using thermoanemometric probe, diploma thesis (2015) 